\title{
Emphysematous Pyelitis With Query Intraparenchymal Involvement
}

Taraneh Tofighi MD, Karen Lien MD, Raphael Rush MD FRCPC

\section{About the Authors}

Taraneh Tofighi, Karen Lien and Raphael Rush are with the Department of Medicine, University of Toronto, Toronto, ON, Canada Corresponding Author: tara.tofighi@mail.utoronto.ca

Submitted: November 9, 2018. Accepted: December 18, 2018. Published: November 19, 2019. DOI: 10.22374/cjgim.v14i4.330

A 65-year-old woman presented with one week of progressive right flank pain radiating to the epigastrium, pneumaturia, and vomiting. Medical history included recurrent urinary tract infections and type 2 diabetes mellitus with hemoglobin A1c of $9.9 \%$. An exam revealed suprapubic tenderness with blood pressure $95 / 60 \mathrm{~mm} \mathrm{Hg}$ and temperature $38.5^{\circ} \mathrm{C}$.

Laboratory investigations revealed leukocytes of 17 (normal 4.3-10.8) $\times$ 109/L. Creatinine was 91 (baseline 70) $\mu \mathrm{mol} / \mathrm{L}$, AST 552 (normal 10-40) U/L and ALT 334 (normal 7-56) U/L. Extended electrolytes revealed serum phosphate 0.35

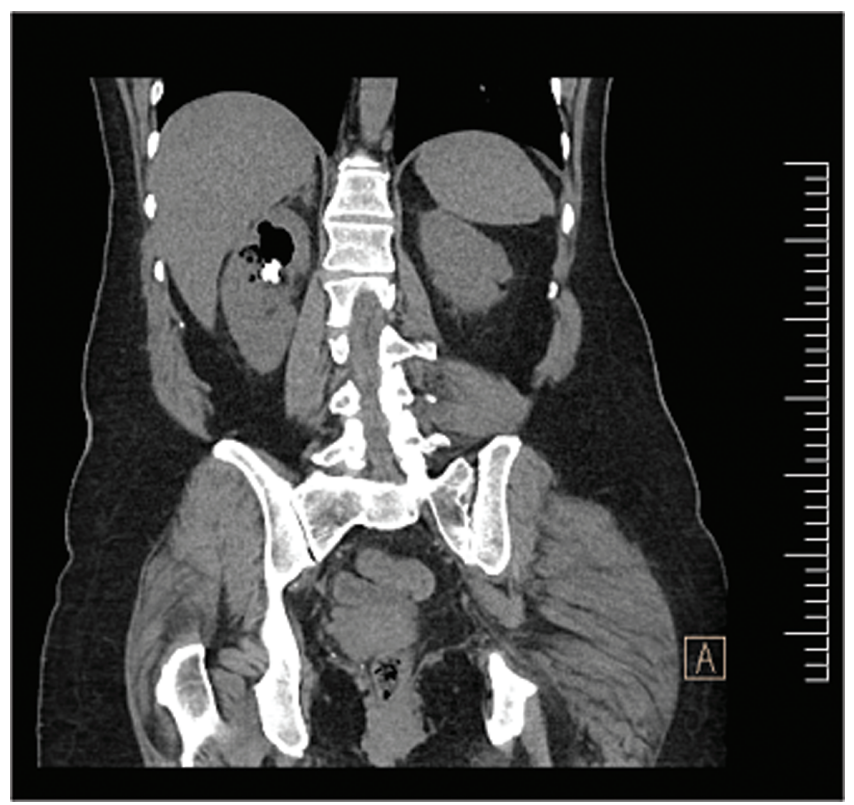

(normal 1.0 to 1.5 ) $\mathrm{mmol} / \mathrm{L}$ and magnesium 0.51 (normal 0.65 to 1.05$) \mathrm{mmol} / \mathrm{L}$ with a lactic acid of $4.2 \mathrm{mmol} / \mathrm{L}$. Computed tomography $(\mathrm{CT})$ revealed emphysematous pyelitis in the right kidney with query parenchymal involvement (Figure 1). Urine culture developed Escherichia coli with negative blood culture. She was managed with crystalloid resuscitation, electrolyte repletion, and antibiotics. Vitals stabilized and lactic acid decreased to $1.9 \mathrm{mmol} / \mathrm{L}$ within 24 hours. Repeat CT revealed an obstructive calculus (Figure 2), with nephrostomy tube placed on day six.

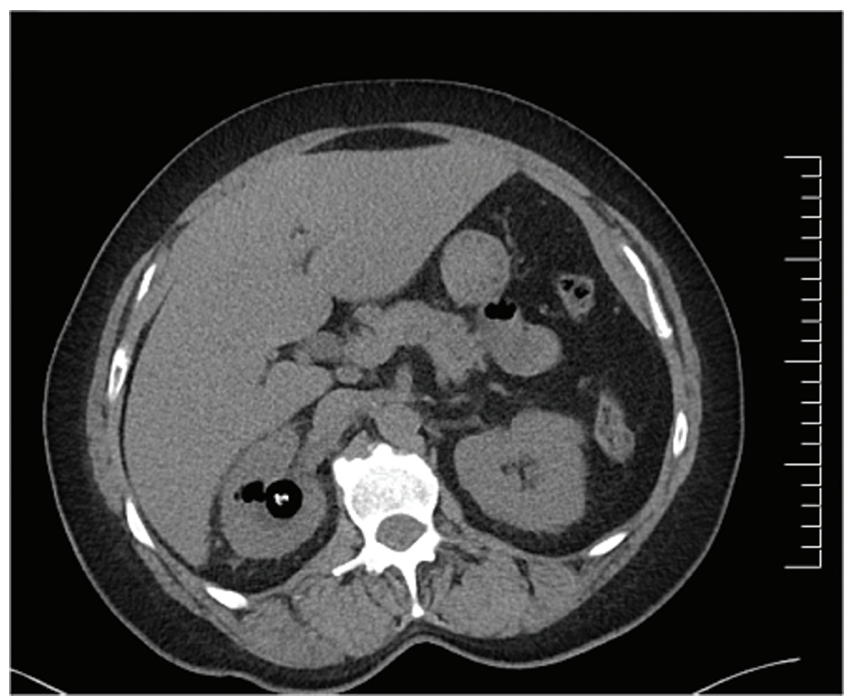

Figure 1. (A) Coronal and (B) Transverse views of the patient's abdomen and pelvis by computed tomography (CT) on presentation revealing emphysematous pyelitis, with possible parenchymal involvement. CT revealed gas in right upstream upper pole calyces extending to right ureter and bladder. 

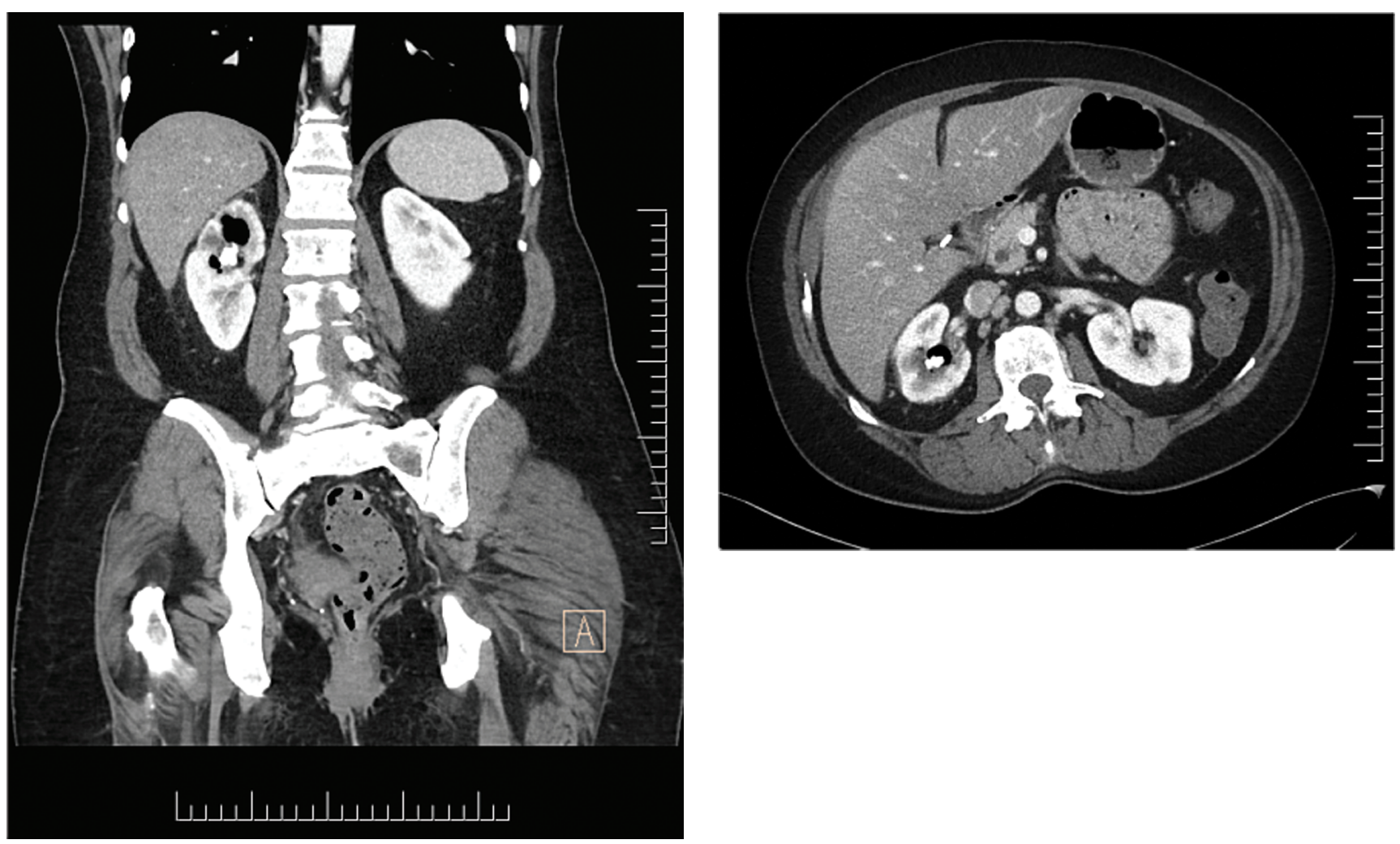

Figure 2. (A) Coronal and (B) Transverse views of the patient's abdomen and pelvis by computed tomography (CT) on day 5 of admission indicating improvement of air and fluid within the obstructed upper pole of the right kidney. There is some loss of cortex in the upper pole of the right kidney and a $1.5 \times 1 \mathrm{~cm}$ calculus obstructing the right upper pole calyceal system.

Emphysematous infections are often associated with diabetes mellitus and can rapidly progress to sepsis, precipitated by glucose-fermenting organisms such as Escherichia coli and Klebsiella. ${ }^{1}$ Clinically, emphysematous pyelitis and the more fulminant emphysematous pyelonephritis (EPN) - characterized by intraparenchymal involvement-are critical to distinguish by CT to guide early management. Emphysematous pyelitis may be managed with antibiotics alone and is associated with a mortality rate of $20 \%$, whereas EPN carries a mortality of $50 \%$, typically requiring surgical drainage or nephrectomy. ${ }^{1,2}$

This case presented a diagnostic challenge in distinguishing emphysematous pyelitis from EPN with CT. In consultation with urology, we elected to conservatively manage the patient given rapid clinical stabilization. Minimally invasive modalities may be successful in the management of emphysematous pyelitis where
EPN is difficult to exclude by radiologic study, with surgical intervention reserved for unresponsive cases.

\section{Funding}

This research did not receive any specific grant from funding agencies in the public, commercial, or not-for-profit sectors.

\section{Conflicts of Interest}

None.

\section{References}

1. Huang J, Tseng C. Emphysematous pyelonephritis. Arch Internal Med 2000;160(6):797.

2. Grayson D, Abbott R, Levy A, et al. Emphysematous infections of the abdomen and pelvis: a pictorial review. RadioGraphics 2002;22(3):543-61. 